\title{
Use of Proton Pump Inhibitors for the Provision of Stress Ulcer Prophylaxis: Clinical and Economic Consequences
}

\author{
Jeffrey F. Barletta - David A. Sclar
}

Published online: 25 November 2013

(C) Springer International Publishing Switzerland 2013

\begin{abstract}
The provision of stress ulcer prophylaxis (SUP) for the prevention of clinically significant bleeding is widely recognized as a crucial component of care in critically ill patients. Nevertheless, SUP is often provided to non-critically ill patients despite a risk for clinically significant bleeding of roughly $0.1 \%$. The overuse of SUP therefore introduces added risks for adverse drug events and cost, with minimal expected benefit in clinical outcome. Historically, histamine-2-receptor antagonists (H2RAs) have been the preferred agent for SUP; however, recent data have revealed proton pump inhibitors (PPIs) as the most common modality $(76 \%)$. There are no high quality randomized controlled trials demonstrating superiority with PPIs compared with H2RAs for the prevention of clinically significant bleeding associated with stress ulcers. In contrast, PPIs have recently been linked to several adverse effects including Clostridium difficile diarrhea and pneumonia. These complications have substantial economic consequences and have a marked impact on the overall cost effectiveness of PPI therapy. Nevertheless, PPI use remains widespread in patients who are at both high and low risk for clinically significant bleeding. This article will describe the utilization of PPIs for SUP and present the clinical and economic consequences linked to their use/ overuse.
\end{abstract}

\section{Key Points for Decision Makers}

- Proton pump inhibitors surpassed histamine-2-receptor antagonists (H2RAs) as the most common agent utilized for the provision of stress ulcer prophylaxis. Several studies have demonstrated excessive use of PPIs in patients at low risk for clinically significant bleeding, in whom the benefit is likely unrecognized and could be associated with significant economic waste.

- There are no high quality randomized controlled trials demonstrating superiority of PPIs versus H2RAs. Several studies have reported a strong association of intense acid suppression with infectious adverse effects such as pneumonia and Clostridium difficile diarrhea. These adverse effects are associated with costs that exceed US $\$ 14,000$ per patient.

- The overall cost effectiveness of PPIs will be based on the incidence of clinically significant bleeding at baseline, the observed reduction in bleeding rates with therapy and the adverse effects that occur with their use. In settings where the incidence of bleeding is low (e.g., non-intensive care unit patients), it is doubtful that PPIs will be cost effective. In settings where the incidence of bleeding is high (e.g., mechanically ventilated patients), clinicians must balance the costs avoided through the prevention of bleeding with the costs accrued through the occurrence of adverse events.

\section{Introduction}

Proton pump inhibitors (PPIs) are widely used for the provision of stress ulcer prophylaxis (SUP) in critically ill patients. In a recent multicenter study, PPIs were selected in $76 \%$ of patients who were administered acid suppressive medications
J. F. Barletta $(\bowtie) \cdot$ D. A. Sclar

Department of Pharmacy Practice, College of Pharmacy-

Glendale, Midwestern University, 19555 N 59th Avenue,

Glendale, AZ 85308, USA

e-mail: jbarle@midwestern.edu 
for SUP compared with $23 \%$ who received histamine-2receptor antagonists (H2RAs) [1]. This is vastly different from surveys published in 2004 and 1999 revealing PPI use for this indication was only 23 and $3 \%$, respectively [2, 3].

Proton pump inhibitors provide more potent acid inhibition than H2RAs, but this has not translated to improvement in clinically significant bleeding in randomized controlled trials. In fact, the evidence base for SUP is extremely limited, outdated and, in many cases, methodologically flawed. This has raised many key questions with regard to the medications used and the overall benefit of SUP. Several studies have actually linked the PPIs with an increased risk for infectious complications such as pneumonia and Clostridium difficile. Additionally, SUP is frequently prescribed to patients with an extremely low risk of clinically important bleeding $(<0.2 \%)$, such as those admitted to a non-intensive care unit (non-ICU) $[4,5]$. The widespread use of PPIs in this setting has the potential to create large increases in healthcare expenditures (through drug acquisition costs and costs related to adverse effects) with minimal benefit in clinical outcome (e.g., a reduction in the incidence of clinically significant bleeding). This article will describe the utilization of PPIs for SUP and present the clinical and economic consequences linked to their use/overuse.

\section{Epidemiology}

Stress-ulcer-related, clinically important bleeding is associated with a significant increase in morbidity and mortality. In one large study of more than 2,200 patients, mortality was $49 \%$ in patients who developed clinically important bleeding compared with $9 \%$ in those without bleeding [4]. A second study reported the relative risk for mortality attributable to clinically important bleeding to range between 1 and 4 (depending on the modeling method) with an increase in hospital length of stay of approximately 4-8 days [6]. This high mortality is the impetus behind the administration of preventative therapy. That being said, the incidence of bleeding is difficult to quantify and varies based on the definition used. The classic definition for clinically important bleeding has been described elsewhere, but variations exist throughout the literature, which muddle the interpretation of the true risk $[4,7,8]$. Nevertheless, the incidence of clinically important bleeding is roughly $0.1-4 \%$, which has decreased substantially over the last 20-30 years [9, 10]. This is largely due to the advances made in resuscitation strategies and therapeutic monitoring.

\section{Risk Factors for Clinically Important Bleeding}

The appropriateness of SUP is largely based on the presence of risk factors for clinically important bleeding. The two risk factors that have been consistently identified in clinical trials are respiratory failure [odds ratio (OR) 15.6] and coagulopathy (OR 4.3) [4]. In fact, when at least one of these risk factors was present, the incidence of bleeding was $3.7 \%$, compared with $0.1 \%$ when they were absent. This translates to a number needed to treat (NNT) of 30 and 900, respectively. Therefore, patients who are either mechanically ventilated or coagulopathic are at high risk for clinically important bleeding and SUP is warranted. If these risk factors are not present, patients can be considered low risk and SUP should be withheld.

A second study aimed to identify additional factors (both predisposing and protective) that were associated with bleeding among a cohort of ICU patients who were mechanically ventilated (therefore considered high risk) [11]. The only independent predictor for bleeding in this study was renal failure. One factor that was identified as being protective was the use of enteral nutrition. This has led many to question if SUP is necessary in patients who receive tube feeds $[12,13]$. While there are no randomized controlled studies directly comparing acid suppressive medications versus tube feeds, one meta-analysis revealed no benefit with SUP in patients who were fed enterally [14]. Future prospective clinical trials are needed in this area.

The risk for stress-ulcer-induced bleeding has also been evaluated in the setting of cardiac surgery. Concerns in this population include the presence of multiple risk factors such as heart failure, use of anticoagulants, cardiopulmonary bypass and prolonged aortic cross-clamping time; all of which can lead to ischemia, the primary etiologic factor for stress-ulcer-induced bleeding [15]. Unfortunately, data specific to cardiac surgery for which clinically important bleeding was the primary endpoint is sparse [16]. In one review, the incidence of bleeding in 42,123 patients who did not receive acid suppressants was only $0.45 \%$ [15]. When acid suppressants were administered ( $n=47,868)$, the incidence of bleeding was $0.35 \%$. Cardiac surgery, therefore, does not independently characterize a patient as being high risk.

There are few studies evaluating the incidence of clinically important bleeding in low-risk patients. This would include a majority of patients who are admitted to a nonICU setting as these patients typically are not coagulopathic or mechanically ventilated. One large study of more than 78,000 admissions (non-ICU) reported the incidence of clinically significant bleeding to be $0.26 \%$ in patients who received acid suppressive medications and $0.18 \%$ in those who did not [5]. This translates to an NNT of 834 to prevent one clinically significant bleed. Interestingly, $81 \%$ of the patients exposed to acid suppressive medications received PPIs. In a follow-up study (using the same database), risk factors for nosocomial gastrointestinal (GI) 
bleeding were identified and a clinical risk score was developed [17]. Factors included were age $>60$ years, male gender, acute renal failure, liver disease, sepsis, prophylactic anticoagulation, coagulopathy and admission to a medicine service. Using this model, the incidence of bleeding in patients not exposed to acid suppressive therapy was $0.04,0.67,1.16$ and $3.24 \%$ when the risk score was $\leq 7,8-9,10-11$ and $\geq 12$, respectively. It should be noted, though, that the model was developed using the endpoint of nosocomial GI bleeding and not clinically important bleeding. Only about $20 \%$ of clinically evident bleeding is clinically significant, which is the gold-standard used in most clinical trials [18]. Nevertheless, the risk for bleeding in the non-ICU setting is extremely low, and it is unlikely that routine acid suppressive therapy will be cost effective.

\section{Clinical Trials Evaluating Proton Pump Inhibitors (PPIs) in High-Risk Patients}

The utilization of PPIs as the acid suppressive medication of first choice is increasing, but the data evaluating their benefit are few and the quality is low. In fact, there are no randomized controlled trials that evaluate intravenous (IV) PPIs and utilize clinically important bleeding as the primary outcome measure. The largest trial was a noninferiority analysis that compared immediate-release omeprazole oral suspension with IV cimetidine in 359 patients [19]. Median gastric pH was significantly higher with omeprazole throughout the study period, but there was no significant difference in the incidence of clinically significant bleeding ( 4.5 vs. $6.8 \%$ ). Similar findings were reported in a second double-blind, randomized controlled trial, which compared omeprazole suspension with IV ranitidine [20]. Despite using a ranitidine dose $(50 \mathrm{mg} \mathrm{Q}$ $12 \mathrm{~h}$ ) that was lower than that recommended in the package insert, there was no difference in the incidence of clinically significant bleeding (1.6 and $5.9 \%$, respectively; $p=$ not significant). A third study compared IV omeprazole with ranitidine in 67 critically ill patients [21]. The incidence of clinically important bleeding was $6 \%$ with omeprazole and $31 \%$ with ranitidine $(p<0.05)$. The study has been criticized, though, because of the uncharacteristically high incidence of bleeding that was reported and the fact that the ranitidine group had a greater number of risk factors for bleeding [8]. Finally, IV omeprazole was compared with famotidine, sucralfate or placebo, and no significant difference in the incidence of clinically significant bleeding was noted $(1,3,4$ and $1 \%$, respectively) [22].

Other studies have compared PPIs with H2RAs in highrisk patients, but interpretation of these studies is difficult because of the use of alternative endpoints (e.g., overt bleeding, gastric $\mathrm{pH}$ ) and high risk of bias. Nevertheless, there have been several meta-analyses that have been published attempting to compare PPIs with H2RAs [7, 8, 23]. The first included 936 patients from seven studies published before May 2008 [8]. Omeprazole was the most common PPI evaluated (IV: three studies; enteral: three studies). The overall pooled risk difference comparing GI bleeding between PPIs and H2RAs was -0.04 [95\% confidence interval (CI) -0.09 to $0.01 ; p=0.08$ ], but significant heterogeneity was noted among the seven included studies. Additionally, only three studies were noted to have good quality. A second meta-analysis included eight fully published trials and five abstracts published between 1993 and $2010(n=1,587$ patients $)$ [23]. Collectively, the overall quality of the included studies was poor, and the definition of clinically significant bleeding varied substantially. Overall, the rates of GI bleeding were lower with PPI therapy than with H2RAs $[$ OR $(95 \% \mathrm{CI})=0.3 \quad(0.17-0.54)]$. Several subgroup analyses were conducted, including variations of the definition for bleeding, publication type (fully published vs. abstract) and date of publication, and in each the original findings remained unchanged. A final meta-analysis included 1,720 patients from 14 trials (four as abstracts) published before March 2012 [7]. Twelve trials reported clinically important bleeding (using multiple definitions), and PPIs were associated with a lower risk compared with H2RAs [risk ratio (RR) $(95 \% \mathrm{CI})=0.36(0.19-0.68$; $p=0.002)]$. A planned subgroup analysis was performed based on the degree of bias of the included studies (three were judged to be a low risk, six were high and five were unclear), and a statistically significant difference was noted between trials having a low risk of bias versus those that were high or unclear. For studies having a low risk of bias, the RR $(95 \% \mathrm{CI})$ for clinically important bleeding was 0.6 (0.27-1.35). With that, clinicians must interpret these results (and the results from other meta-analyses) with caution. Although the meta-analysis itself was conducted with strong scientific rigor, the methodologic quality of the included studies, overall, was poor. There is a strong degree of variability noted within the trials, including differences in the definition for bleeding, the patient population studied, severity of illness and risk factors present and medication regimens utilized. Additionally, the impact of enteral nutrition could not be completely assessed as no trials stratified randomization on the basis of nutritional strategy. Large, randomized controlled trials are needed to provide more definitive guidance regarding the agent of choice, especially given the prior inconsistencies noted between well conducted randomized controlled trials and meta-analyses that compared H2RAs with sucralfate [24, 25]. 
Recommendations for the provision of SUP do exist in evidence-based guidelines. The first guidelines were published in 1999 from the American Society of Health-System Pharmacists. At the time of writing, however, there were insufficient data regarding PPIs to make a recommendation for SUP [9]. Suggestions for a specific agent as per these guidelines are out-dated. The Eastern Association for the Surgery of Trauma developed guidelines for SUP in trauma patients in 2008 and stated there is no difference between H2RAs and PPIs (level I recommendation) [26]. Most recently, the Surviving Sepsis Campaign guidelines suggested the use of PPIs rather than H2RA for SUP; this was a grade $2 \mathrm{C}$ recommendation (i.e., weak/low quality of evidence) [27]. Guidelines from the Society of Critical Care Medicine are currently in development and anticipated to be released shortly.

\section{Overuse of PPIs in Low-Risk Patients}

There are no clinical trials demonstrating benefit with PPI therapy for SUP in low-risk patients (i.e., those outside the ICU). Nevertheless, the utilization of PPIs for this indication is widespread. Survey data (of level I trauma centers and ICU pharmacists) reveal that up to $40 \%$ of respondents administer SUP in low-risk, non-ICU patients more than $50 \%$ of the time $[3,28]$. Retrospective analyses have reported the incidence of inappropriate therapy in low-risk patients (e.g., those outside the ICU) to be roughly 50-60\% [29-34].

The continuation of SUP post-ICU discharge remains problematic (when patients are no longer high risk), and some studies have reported the continuation of SUP beyond hospital discharge. One study of critically ill patients reported that $60 \%$ were transferred out of the ICU on SUP and $31 \%$ continued prophylaxis upon hospital discharge [35]. This is similar to other reports where the continuation of SUP at discharge ranged from 18 to $34 \%$ [34, 36-38]. Leri et al. [39] used the Blue Cross of Northeastern Pennsylvania medical/prescription database to evaluate the number of patients who were discharged from the hospital on a PPI without an indication. Over a 4-year period (January 2005-December 2008), of the 2,686 patients who were discharged on a PPI, $80 \%$ of the PPI prescriptions were deemed inappropriate. The estimated 4-year cost (using drug acquisition costs of US\$3/dose) associated with inappropriate PPI use was US\$595,809.

The vast overuse of SUP in low-risk patients is difficult to explain and likely multifactorial. One potential reason is a lack of concern for serious adverse drug events (ADEs) and their respective clinical and economic sequelae. In one survey of internal medicine physicians, $<50 \%$ stated they were concerned about side effects when considering prescribing acid suppressive therapy for prophylaxis [40]. A second survey revealed that $28 \%$ of attending physicians felt PPIs were "harmless" [41]. Without an appreciation for the adverse effects associated with these agents and their resultant costs, clinicians are likely to continue with these inappropriate practices because of false perceptions of risk and overall cost of care.

\section{Adverse Effects of PPIs}

Randomized controlled trials evaluating PPIs for SUP have not noted a large number of adverse events compared with alternative therapy (e.g., H2RAs), but several ADEs have been noted with PPIs in other settings. The most worrisome are infectious and include pneumonia and $C$. difficile [42, 43]. Acutely, these ADEs appear to be related to the ability of PPIs to increase gastric $\mathrm{pH}$ and allow for bacterial overgrowth, but other mechanisms have been postulated [44].

Infectious complications with PPIs have been well documented in the outpatient setting, and inpatient data are beginning to accumulate [42, 43, 45-50] One large prospective cohort study of non-ICU patients examined the relationship between acid suppressive therapy and hospitalacquired pneumonia [42]. Approximately 64,000 patients were included and $52 \%$ received acid suppressive therapy. PPIs were utilized in a majority of patients (83\%), and in most instances (89\%), medications were ordered within 48 $\mathrm{h}$ of admission. After adjusting for potential confounding variables, acid suppressive therapy was associated with a $30 \%$ increased odds of hospital-acquired pneumonia. A subgroup analysis revealed this risk to be primarily related to PPI use [OR $(95 \% \mathrm{CI})=1.3(1.1-1.4)]$ and not H2RAs $[\mathrm{OR}(95 \% \mathrm{CI})=1.2(0.98-1.4)]$. While temporal information related to the time to diagnosis could not be assessed in this study, extrapolations from the community setting indicate the risk appears to be greatest during the early part of therapy ( $\leq 7$ days) [47]. A second study evaluated the risk of nosocomial pneumonia in cardiac surgery patients [51]. In this study, 21,214 patients who underwent coronary artery bypass graft surgery received either a PPI $(n=9,830)$ or an H2RA $(n=11,384)$. After propensity score adjustment, an elevated risk for pneumonia was noted with PPIs compared with H2RAs [RR $(95 \% \mathrm{CI})=1.19(1.03-1.38)]$.

Proton pump inhibitors have also been associated with C. difficile infection. One study evaluated the impact of increasing levels of pharmacologic acid suppression with nosocomial $C$. difficile infection [43]. In this study, the OR $(95 \% \mathrm{CI})$ for $C$. difficile acquisition increased from 1 (reference $=$ no acid suppression) to 1.53 (1.12-2.1) for H2RAs, 1.74 (1.39-2.18) for daily PPIs and 2.36 
(1.79-3.11) for PPI use more frequent than once daily. A second study revealed that not only the intensity of acid suppression was linked to $C$. difficile, but also the duration of acid suppression, specifically with PPIs [45]. In a matched, case-control study, duration of PPI therapy was identified as an independent predictor for $C$. difficile infection [OR $(95 \% \mathrm{CI})=1.14(1.02-1.27), p=0.018$ ]. Furthermore, thresholds for duration of therapy were identified whereby the likelihood of $C$. difficile increased. In patients who did not have a prior hospital admission within 30 days, the risk for $C$. difficile increased when PPI therapy exceeded 2 days and 12 days. For patients who were previously admitted within 30 days, that threshold was 1 day. Given the short exposure required for the development of both pneumonia and $C$. difficile infection, injudicious prescribing can contribute significantly to the morbidity of hospitalized patients and the overall cost of care.

Other adverse effects described with PPIs include fractures and osteoporosis [52]. These ADEs are more prominent with chronic use and have not been described in studies evaluating SUP. Nevertheless, given the large percentage of patients inadvertently discharged on SUP, this risk cannot be ignored. In one study, the risk was evident even after 1 year of therapy [OR $(95 \% \mathrm{CI})=1.22$ (1.15-1.30)] [52]. Another study reported higher 1-year mortality [hazard ratio $(95 \% \mathrm{CI})=2.59(1.22-7.16)]$ in elderly patients who received PPI therapy at doses commonly used for SUP (e.g., pantoprazole $40 \mathrm{mg}$ daily, lansoprazole $30 \mathrm{mg}$ daily) [53]. The mechanism surrounding this association is likely multifactorial.

\section{Economic Considerations}

\subsection{Outcome-Related Costs}

The overall cost effectiveness of PPIs for SUP is largely based on the costs associated with clinically important bleeding and adverse effects, namely pneumonia and $C$. difficile. The costs associated with upper GI bleeding have been thoroughly evaluated, but most data originate from studies whereby bleeding was the primary diagnosis and reason for the hospital admission [54]. It is questionable if these data can be extrapolated to the inpatient setting where GI bleeding is a secondary complication because of differences in etiology, comorbidities and underlying reason for the index admission. There are few studies specific to stress-ulcer-induced, clinically significant bleeding encountered in the ICU, and in those studies, a wide range of costs exists. This could be related to inflation rates and the year these studies were conducted, but variability in the severity of bleeding, the interventions required and the resultant effects on length of stay can also be considered. For example, costs will be markedly different for a bleeding event that simply requires an increased level of monitoring versus one that necessitates blood transfusions or even a surgical procedure. Ultimately, the driving force behind the costs associated with clinically important bleeding in the ICU are whether or not the event leads to an increase in ICU length of stay.

In one small matched cohort study, clinically important bleeding was associated with a trend towards an increased ICU length of stay of 6.5 days (95 \% CI -12.3 to 25.3) [55]. The overall costs were Canadian dollars (Can\$) 12,215 (1993 year value). A follow-up study was conducted by the same investigators to better estimate the attributable outcomes related to clinically important bleeding [6]. There were 1,666 mechanically ventilated patients evaluated using three different analytical techniques. Overall, ICU length of stay increased by 4-8 days when clinically important bleeding occurred. Barkun et al. [56] conducted an economic evaluation from a third-party payer perspective to quantify the cost effectiveness of PPIs versus H2RAs for SUP. A decision-tree model was constructed with the terminal node being cost per no complication; complications included GI bleeding and ventilator associated pneumonia (VAP). The underlying costs and length of stay resultant to these complications were obtained from the National Inpatient Sample (2008) and relevant ICD-9 codes. There were no differences noted in the per-diem cost between patients with no complications (US\$2,993) compared with those with GI bleeding (US\$2,764) and VAP (US\$3,310) (2010 year values). Length of stay, however, was 14 days in patients with no complications, 24 days in patients with GI bleeding and 42 days in patients with VAP. Overall, this translated to an increase in costs of roughly US\$24,000 and US\$96,100 when GI bleeding and VAP occurred, respectively.

Other investigators have described the costs associated with pneumonia. One study utilized administrative claims from the Centers for Medicare and Medicaid Service Chronic Condition Warehouse (2005-2007 for analyses and 2004 for baseline data). Patients with pneumonia were matched to a cohort of control patients (without pneumonia) using propensity score matching, and costs were captured over a 2-year period. Patients with pneumonia observed excess costs of US\$13,632 (2005 year value) in the first 3 months post-diagnosis compared with matched controls without pneumonia [57]. The total difference in costs for the 2-year period was US\$16,132. A second study utilized the discharge records from the 1998-2006 National Inpatient Sample database to assess clinical and economic outcomes attributable to healthcare-associated infections. Patients with hospital acquired infections were matched to patients without infections using several variables such as 
primary diagnosis, principal procedure, age, comorbidities, urgency of admission and year of discharge. Overall, the attributable costs (2006 year values) associated with the development of pneumonia were US\$22,300 in non-surgical patients and US $\$ 46,400$ for those who underwent surgical procedures [58]. This economic burden may be even higher in patients with infections caused by antimicrobial-resistant organisms. One study compared hospital charges in patients who developed healthcare-associated infections between 2006 and 2008 from four hospitals in metropolitan New York City [59]. Using linear regression and nearest neighbor matching based on propensity score estimates, resistant infections were associated with significantly higher charges (US\$15,626; CI 4,339-26,913) when compared with patients with infections caused by a susceptible strain of the same organism.

The economic consequences of $C$. difficile have also been reported. One study examined hospitalizations for $C$. difficile during 2001-2002 using Massachusetts hospital discharge databases. Patients were identified using the ICD-9 code (008.45) and classified as having either primary or secondary $C$. difficile. Patients with secondary $C$. difficile (i.e., acquired in the hospital) had a mean cost per stay of US\$29,946 (2005 year value). Based on the increase in length of stay observed in patients with $C$. difficile compared with those without (2.9 days), it was determined the mean cost attributable to $C$. difficile per stay was US $\$ 13,675$ [60]. As such, the cost of a hospital stay would be $46 \%$ lower for patients who did not acquire $C$. difficile-associated diarrhea.

Other economic considerations include the costs associated with inadvertently continuing PPI therapy upon discharge. While infectious complications like pneumonia and $C$. difficile appear to occur early in the course of therapy, others like the increase in fracture risk are related to chronic therapy. Nevertheless, these complications are extremely costly to the healthcare system and are associated with significant morbidity. Processes like medication reconciliation are designed to minimize unnecessary therapies across the continuum of care, but these methods appear minimally effective with regard to PPI therapy and SUP [38].

Given the costly consequences associated with therapy (both positive and negative), the overall cost effectiveness of PPIs for SUP will be determined by the NNT to prevent a clinically important bleeding event and the number needed to harm (NNH) by an adverse event. For example, in high-risk patients, such as a critically ill patient with respiratory failure receiving mechanical ventilation, the NNT is approximately 30 [4]. Conversely, in a low-risk patient, like a patient admitted to a medical/surgical floor following elective surgery, the NNT is 834 [5]. This must be balanced by the NNH encompassing all ADEs. Based on the aforementioned data, the $\mathrm{NNH}$ with pneumonia is approximately 111 and the NNH with $C$. difficile is 533 $[42,43]$. Further research is needed to generate additional clinical data (in both the ICU and non-ICU settings) that can further elucidate benefits and risks with acid suppressive therapies. These data can then be used in pharmacoeconomic analyses to determine the overall cost of therapy when all outcomes are intertwined and identify the most cost-effective option.

\subsection{Published Pharmacoeconomic Analyses}

Economic evaluations of SUP and PPI therapy have been reported using various techniques [56, 61, 62]. One report examined the practice of SUP in non-ICU patients, with a specific emphasis on resource utilization [61]. Consecutive admissions to a large academic teaching hospital over a 4-month period were retrospectively evaluated to quantify the utilization of acid suppressive medications for SUP. Economic impact was assessed using cost data based on the number of pills administered, inclusive of inpatient use and 30-day outpatient prescriptions if applicable, and 2004 average wholesale price (AWP) data. Pantoprazole was the most frequent medication used (89\%), and inpatient costs associated with unnecessary SUP totaled US $\$ 11,024$ for the 4-month study period. Outpatient pharmacy costs (based on prescriptions given at discharge) were US $\$ 16,924$. Annualized, these represent a burden of US $\$ 111,791$ to the healthcare system. It is likely, though, that these data markedly underestimate the true burden to the healthcare system given that adverse effects and outcomes were not factored into this analysis.

Udeh et al. [62] conducted a cost-effectiveness analysis using various PPI regimens, famotidine and sucralfate for SUP. A decision-tree model was constructed from the perspective of high-risk patients, and the cost per bleeding event avoided was computed for seven different treatment arms and no therapy. Factors included in the decision tree were 2005 drug acquisition costs (using AWP), consumables and labor costs, costs associated with clinically significant bleeding and costs to evaluate diarrhea, thrombocytopenia and mental status changes (obtained from the American Society of Health-System Pharmacists guidelines). The option that was most cost effective was SUP using an enteral PPI (i.e., immediate-release omeprazole) at US $\$ 12,391$ per bleeding event avoided. This was the first pharmacoeconomic analysis that assessed PPI therapy and accounted for ADEs. However, the costs that were used to represent adverse effects were not consistent with those reported in other pharmacoeconomic studies. For example, the cost estimate used for diarrhea was only US\$219, which is substantially lower than the cost reported for diarrhea caused by $C$. difficile in other studies. 
Additionally, the exclusion of pneumonia in the model can be questioned.

Finally, a third economic evaluation compared PPIs with H2RAs in the setting of high-risk patients in the ICU from a third-party payer perspective [56]. In this study, a decision-tree model was constructed that included hospital costs stratified by medication (PPI vs. H2RA) and the occurrence of a complication (i.e., bleeding, pneumonia). Resultant length of stay and hospital costs were obtained from the Nationwide Inpatient Sample 2008 and relevant ICD-9 codes. The incidence of bleeding and pneumonia were determined using the published literature and were estimated to be $1.34 \%$ (PPI) and $6.61 \%$ (H2RA) for bleeding and $10.33 \%$ (PPI) and $10.32 \%$ (H2RA) for pneumonia. In this analysis, PPIs were the most cost effective (average costs per no complication [2010 year values] US\$58,700 vs. US\$63,920). These findings were sensitive to the incidence of pneumonia. Costs associated with $C$. difficile diarrhea were not included in this design.

Despite these analyses, there are several questions that remain. The impact of $C$. difficile as a complication of PPI therapy is being increasingly noted. How severely this will influence a pharmacoeconomic model is undetermined. Additionally, the available comparative analyses address PPI use primarily in high-risk patients. The comprehensive economic burden that exists when low-risk patients are accounted for has not been quantified. As institutions develop guidelines that might recommend PPI therapy, they must consider the potential for therapy to infiltrate the non-ICU setting and the economic consequences that ensue. Finally, the continued use of PPI therapy in the outpatient setting remains problematic. The outcomes that follow and their resultant costs will require further observation.

\section{Conclusions}

Proton pump inhibitors are increasingly being used as the primary agent to provide SUP in critically ill patients. Furthermore, these agents are often initiated (or continued) in non-ICU patients where the risk of clinically important bleeding is minuscule and the benefit of such therapy is lacking. The clinical data evaluating PPIs for SUP is of low quality and most studies were conducted in an era where practices differ tremendously from today's approach. The value of meta-analyses in this setting is limited, and large, multicenter, randomized controlled trials are needed. Given the fact that economic consequences of PPI use for SUP are largely driven by outcome (clinically important bleeding) and adverse effects (infectious complications), the risk for pneumonia and $C$. difficile must be appreciated in future economic analyses.
Acknowledgments Jeffrey F. Barletta has no conflicts of interest to report. David A. Sclar has received grant funding from Optimer Pharmaceuticals. Drs. Barletta and Sclar were both involved in the conception of the idea for the review, drafting the original manuscript and making the appropriate edits during the review process. Dr. Barletta is the overall guarantor of accuracy for the manuscript.

\section{References}

1. Barletta JF, Lat I, Micek S, Cohen H, Olsen K, Haas C. Off label use of gastrointestinal medications in the intensive care unit. J Intensive Care Med. 2011 (in press).

2. Daley RJ, Rebuck JA, Welage LS, Rogers FB. Prevention of stress ulceration: current trends in critical care. Crit Care Med. 2004;32(10):2008-13.

3. Erstad BL, Barletta JF, Jacobi J, Killian AD, Kramer KM, Martin SJ. Survey of stress ulcer prophylaxis. Critical care (London, England). 1999;3(6):145-9.

4. Cook DJ, Fuller HD, Guyatt GH, Marshall JC, Leasa D, Hall R, et al. Risk factors for gastrointestinal bleeding in critically ill patients. Canadian Critical Care Trials Group. N Engl J Med. 1994:330(6):377-81.

5. Herzig SJ, Vaughn BP, Howell MD, Ngo LH, Marcantonio ER. Acid-suppressive medication use and the risk for nosocomial gastrointestinal tract bleeding. Arch Intern Med. 2011;171(11):991-7.

6. Cook DJ, Griffith LE, Walter SD, Guyatt GH, Meade MO, Heyland DK, et al. Critical care (London, England). 75. 2001;5(6):368-75.

7. Alhazzani W, Alenezi F, Jaeschke RZ, Moayyedi P, Cook DJ. Proton pump inhibitors versus histamine 2 receptor antagonists for stress ulcer prophylaxis in critically ill patients: a systematic review and meta-analysis. Crit Care Med. 2013;41(3):693-705.

8. Lin PC, Chang CH, Hsu PI, Tseng PL, Huang YB. The efficacy and safety of proton pump inhibitors vs histamine-2 receptor antagonists for stress ulcer bleeding prophylaxis among critical care patients: a meta-analysis. Crit Care Med. 2010;38(4):1197-205.

9. ASHP Therapeutic Guidelines on Stress Ulcer Prophylaxis. ASHP Commission on Therapeutics and approved by the ASHP Board of Directors on November 14, 1998. Am. J Health Syst Pharm. 1999;56(4):347-79.

10. Krag M, Perner A, Wetterslev J, Moller MH. Stress ulcer prophylaxis in the intensive care unit: is it indicated? A topical systematic review. Acta Anaesthesiologica Scandinavica. 2013;57(7):835-47.

11. Cook D, Heyland D, Griffith L, Cook R, Marshall J, Pagliarello J. Risk factors for clinically important upper gastrointestinal bleeding in patients requiring mechanical ventilation. Canadian Critical Care Trials Group. Crit Care Med. 1999;27(12):2812-7.

12. Hurt RT, Frazier TH, McClave SA, Crittenden NE, Kulisek C, Saad M, et al. Stress prophylaxis in intensive care unit patients and the role of enteral nutrition. JPEN J Parent Enter Nutr. 2012;36(6):721-31.

13. Marik PE. Stress ulcer prophylaxis in the new millennium! ICU Dir. 2010;1(1):12-6.

14. Marik PE, Vasu T, Hirani A, Pachinburavan M. Stress ulcer prophylaxis in the new millennium: a systematic review and meta-analysis. Crit Care Med. 2010;38(11):2222-8.

15. van der Voort PH, Zandstra DF. Pathogenesis, risk factors, and incidence of upper gastrointestinal bleeding after cardiac surgery: is specific prophylaxis in routine bypass procedures needed? J Cardiothorac Vasc Anesth. 2000;14(3):293-9.

16. Rosen HR, Vlahakes GJ, Rattner DW. Fulminant peptic ulcer disease in cardiac surgical patients: pathogenesis, prevention, and management. Crit Care Med. 1992;20(3):354-9. 
17. Herzig SJ, Rothberg MB, Feinbloom DB, Howell MD, Ho KK, Ngo LH, et al. Risk factors for nosocomial gastrointestinal bleeding and use of acid-suppressive medication in non-critically ill patients. J Gen Intern Med. 2013;28(5):683-90.

18. Ali T, Harty RF. Stress-induced ulcer bleeding in critically ill patients. Gastroenterol Clin N Am. 2009;38(2):245-65.

19. Conrad SA, Gabrielli A, Margolis B, Quartin A, Hata JS, Frank WO, et al. Randomized, double-blind comparison of immediaterelease omeprazole oral suspension versus intravenous cimetidine for the prevention of upper gastrointestinal bleeding in critically ill patients. Crit Care Med. 2005;33(4):760-5.

20. Solouki M, Marashian S, Kouchak M. Comparison between the preventive effects of ranitidine and omeprazole on upper gastrointestinal bleeding among ICU patients. Tanaffos. 2009;8:37-42.

21. Levy MJ, Seelig CB, Robinson NJ, Ranney JE. Comparison of omeprazole and ranitidine for stress ulcer prophylaxis. Dig Dis Sci. 1997;42(6):1255-9.

22. Kantorova I, Svoboda P, Scheer P, Doubek J, Rehorkova D, Bosakova $\mathrm{H}$, et al. Stress ulcer prophylaxis in critically ill patients: a randomized controlled trial. Hepato Gastroenterol. 2004;51(57):757-61.

23. Barkun AN, Bardou M, Pham CQ, Martel M. Proton pump inhibitors vs. histamine 2 receptor antagonists for stress-related mucosal bleeding prophylaxis in critically ill patients: a metaanalysis. Am J Gastroenterol. 2012;107(4):507-20 (quiz 21).

24. Cook D, Guyatt G, Marshall J, Leasa D, Fuller H, Hall R, et al. A comparison of sucralfate and ranitidine for the prevention of upper gastrointestinal bleeding in patients requiring mechanical ventilation. Canadian Critical Care Trials Group. N Engl J Med. 1998;338(12):791-7.

25. Cook DJ, Reeve BK, Guyatt GH, Heyland DK, Griffith LE, Buckingham L, et al. Stress ulcer prophylaxis in critically ill patients. Resolving discordant meta-analyses. JAMA. 1996;275(4):308-14.

26. Guillamondegui OD, Gunter Jr OL, Bonadies JA, Coates JE, Kurek SJ, DeMoya MA, et al. Practice management guidelines for stress ulcer prophylaxis. EAST Practice Management Guidelines Committee. http://wwweastorg/resources/treatmentguidelines/stress-ulcer-prophylaxis (2008). Accessed 3 July 2013.

27. Dellinger RP, Levy MM, Rhodes A, Annane D, Gerlach H, Opal SM, et al. Surviving sepsis campaign: international guidelines for management of severe sepsis and septic shock: 2012. Crit Care Med. 2013;41(2):580-637.

28. Barletta JF, Erstad BL, Fortune JB. Stress ulcer prophylaxis in trauma patients. Critical care (London, England). 2002;6(6):526-30.

29. Gupta R, Garg P, Kottoor R, Munoz JC, Jamal MM, Lambiase $\mathrm{LR}$, et al. Overuse of acid suppression therapy in hospitalized patients. South Med J. 2010;103(3):207-11.

30. Issa IA, Soubra O, Nakkash H, Soubra L. Variables associated with stress ulcer prophylaxis misuse: a retrospective analysis. Dig Dis Sci. 2012;57(10):2633-41.

31. Judd WR, Davis GA, Winstead PS, Steinke DT, Clifford TM, Macaulay TE. Evaluation of continuation of stress ulcer prophylaxis at hospital discharge. Hosp Pharm. 2009;44:888-93.

32. Nardino RJ, Vender RJ, Herbert PN. Overuse of acid-suppressive therapy in hospitalized patients. Am J Gastroenterol. 2000; 95(11):3118-22.

33. Parente F, Cucino C, Gallus S, Bargiggia S, Greco S, Pastore L, et al. Hospital use of acid-suppressive medications and its fall-out on prescribing in general practice: a 1-month survey. Aliment Pharmacol Therap. 2003;17(12):1503-6.

34. Zink DA, Pohlman M, Barnes M, Cannon ME. Long-term use of acid suppression started inappropriately during hospitalization. Aliment Pharmacol Therap. 2005;21(10):1203-9.
35. Farrell CP, Mercogliano G, Kuntz CL. Overuse of stress ulcer prophylaxis in the critical care setting and beyond. J Crit Care. 2010;25(2):214-20.

36. Murphy CE, Stevens AM, Ferrentino N, Crookes BA, Hebert JC, Freiburg $\mathrm{CB}$, et al. Frequency of inappropriate continuation of acid suppressive therapy after discharge in patients who began therapy in the surgical intensive care unit. Pharmacotherapy. 2008;28(8):968-76.

37. Wohlt PD, Hansen LA, Fish JT. Inappropriate continuation of stress ulcer prophylactic therapy after discharge. Ann Pharmacother. 2007;41(10):1611-6.

38. Zeigler AJ, McAllen KJ, Slot MG, Barletta JF. Medication reconciliation effect on prolonged inpatient stress ulcer prophylaxis. Ann Pharmacother. 2008;42(7):940-6.

39. Leri F, Ayzenberg M, Voyce SJ, Klein A, Hartz L, Smego RA Jr. Four-year trends of inappropriate proton pump inhibitor use after hospital discharge. South Med J. 2013;106(4):270-3.

40. Hussain S, Stefan M, Visintainer P, Rothberg M. Why do physicians prescribe stress ulcer prophylaxis to general medicine patients? South Med J. 2010;103(11):1103-10.

41. Koczka CP, Geraldino-Pardilla LB, Goodman AJ. Physicians' opinions of stress ulcer prophylaxis: survey results from a large urban medical center. Dig Dis Sci. 2013;58(3):777-81.

42. Herzig SJ, Howell MD, Ngo LH, Marcantonio ER. Acid-suppressive medication use and the risk for hospital-acquired pneumonia. JAMA. 2009;301(20):2120-8.

43. Howell MD, Novack V, Grgurich P, Soulliard D, Novack L, Pencina $\mathrm{M}$, et al. Iatrogenic gastric acid suppression and the risk of nosocomial Clostridium difficile infection. Arch Intern Med. 2010;170(9):784-90.

44. Meijvis SC, Cornips MC, Voorn GP, Souverein PC, Endeman H, Biesma DH, et al. Microbial evaluation of proton-pump inhibitors and the risk of pneumonia. Eur Resp J. 2011;38(5):1165-72.

45. Barletta JF, El-Ibiary SY, Davis LE, Nguyen B, Raney CR. Proton pump inhibitors and the risk for hospital-acquired Clostridium difficile infection. Mayo Clin Proc. 2013;88(10):1085-90.

46. Dial S, Delaney JA, Barkun AN, Suissa S. Use of gastric acid-suppressive agents and the risk of community-acquired Clostridium difficile-associated disease. JAMA. 2005;294(23): 2989-95.

47. Gulmez SE, Holm A, Frederiksen H, Jensen TG, Pedersen C, Hallas J. Use of proton pump inhibitors and the risk of community-acquired pneumonia: a population-based case-control study. Arch Intern Med. 2007;167(9):950-5.

48. Laheij RJ, Sturkenboom MC, Hassing RJ, Dieleman J, Stricker BH, Jansen JB. Risk of community-acquired pneumonia and use of gastric acid-suppressive drugs. JAMA. 2004;292(16):1955-60.

49. Loo VG, Bourgault AM, Poirier L, Lamothe F, Michaud S, Turgeon $\mathrm{N}$, et al. Host and pathogen factors for Clostridium difficile infection and colonization. N Engl J Med. 2011;365(18): 1693-703.

50. Sarkar M, Hennessy S, Yang YX. Proton-pump inhibitor use and the risk for community-acquired pneumonia. Ann Intern Med. 2008;149(6):391-8.

51. Bateman BT, Bykov K, Choudhry NK, Schneeweiss S, Gagne JJ, Polinski JM, et al. Type of stress ulcer prophylaxis and risk of nosocomial pneumonia in cardiac surgical patients: cohort study. BMJ (Clinical research ed). 2013;347:f5416.

52. Yang YX, Lewis JD, Epstein S, Metz DC. Long-term proton pump inhibitor therapy and risk of hip fracture. JAMA. 2006;296(24):2947-53.

53. Maggio M, Corsonello A, Ceda GP, Cattabiani C, Lauretani F, Butto V, et al. Proton pump inhibitors and risk of 1-year mortality and rehospitalization in older patients discharged from acute care hospitals. JAMA Intern Med. 2013;173(7):518-23. 
54. Parker DR, Luo X, Jalbert JJ, Assaf AR. Impact of upper and lower gastrointestinal blood loss on healthcare utilization and costs: a systematic review. J Med Econ. 2011;14(3):279-87.

55. Heyland D, Gafni A, Griffith L, Cook D, Marshall J, Fuller H, et al. The clinical and economic consequences of clinically important gastro-intestinal bleeding in critically ill patients. Clin Intensive Care. 1996;7:121-5.

56. Barkun AN, Adam V, Martel M, Bardou M. Cost-effectiveness analysis: stress ulcer bleeding prophylaxis with proton pump inhibitors, H2 receptor antagonists. Value Health. 2013;16(1): 14-22.

57. Thomas CP, Ryan M, Chapman JD, Stason WB, Tompkins CP, Suaya JA, et al. Incidence and cost of pneumonia in Medicare beneficiaries. Chest. 2012;142(4):973-81.

58. Eber MR, Laxminarayan R, Perencevich EN, Malani A. Clinical and economic outcomes attributable to health care-associated sepsis and pneumonia. Arch Intern Med. 2010;170(4):347-53.
59. Neidell MJ, Cohen B, Furuya Y, Hill J, Jeon CY, Glied S, et al. Costs of healthcare- and community-associated infections with antimicrobial-resistant versus antimicrobial-susceptible organisms. Clin Infect Dis. 2012;55(6):807-15.

60. O'Brien JA, Lahue BJ, Caro JJ, Davidson DM. The emerging infectious challenge of Clostridium difficile-associated disease in Massachusetts hospitals: clinical and economic consequences. Infect Control Hosp Epidemiol. 2007;28(11):1219-27.

61. Heidelbaugh JJ, Inadomi JM. Magnitude and economic impact of inappropriate use of stress ulcer prophylaxis in non-ICU hospitalized patients. Am J Gastroenterol. 2006;101(10):2200-5.

62. Udeh BL, Udeh C, Hata JS. Cost-effectiveness of stress ulcer prophylaxis: role of proton pump inhibitors. Am J Pharm Benefits. 2010;2(5):304-12. 